\title{
Unidad Nefrológica de Atención Continuada. Experiencia de un Hospital Universitario
}

\author{
Verónica Sánchez Rodrigo, Sonia Mendoza Mendoza, Esther García García, Laura Baena Ruiz, Cristina Pérez \\ Ramos, Jessica López Salas, Esther Fernández Vega
}

Sección de Nefrología. Hospital Universitario del Henares. Coslada. Madrid. España

\section{Resumen}

Introducción: Los hospitales de día suponen una alternativa asistencial a la hospitalización convencional mejorando la eficacia de la asistencia sanitaria. Desde nuestro punto de vista, la nefrología se beneficiaria de esta modalidad de atención, ya que el paciente con enfermedad renal crónica tiene unas necesidades concretas de cuidado que conllevan al profesional de enfermería a generar una atención integral e individualizada. Por este motivo surge en nuestro servicio la creación de la Unidad Nefrológica de Atención Continuada.

Objetivo: Presentar nuestra experiencia en la implantación y desarrollo de la Unidad Nefrológica de Atención Continuada en el Hospital Universitario del Henares.

Material y Método: Estudio descriptivo retrospectivo de la actividad realizada en la Unidad Nefrológica de Atención Continuada entre enero-junio de 2017. Desarrollo y puesta en marcha de las distintas funciones y competencias profesionales.

Resultados: En este periodo se asistieron a un total de 874 pacientes, con una media de 145,6 visitas/mes. De todas estas visitas, se atendieron 474 pacientes en programa de Diálisis Peritoneal ( $55 \%$ del volumen total), 149 pacientes con enfermedad renal crónica (16\%), 245 pacientes con enfermedad renal crónica avanza-

Correspondencia:

Verónica Sánchez Rodrigo

Avenida Marie Curie, 2, 28822 Coslada. Madrid

E-mail: veroquiti@hotmail.com da (18\%) y 11 pacientes en tratamiento conservador ( $1 \%)$. Se realizaron diferentes técnicas, bien programadas en la agenda electrónica 0 a demanda.

Conclusiones: La implantación de la Unidad Nefrológica de Atención Continuada, de forma estructurada y planificada, es una alternativa válida y necesaria en nuestro sistema sanitario. Permite aseguramos un abordaje integral de los pacientes renales y alcanzar una continuidad de cuidados con menor coste sanitario.

PALABRAS CLAVE: fallo renal crónico; atención ambulatoria; centro de atención diurna; enfermería en nefrología.

\section{Nephrological Unit for Continued Care. Experience of a University Hospital}

\section{Abstract}

Introduction: The daytime hospitals are an alternative to conventional hospitalization and improve the effectiveness of healthcare. From our point of view, nephrology is a specialty that should be able to benefit from this type of care, because chronic kidney disease (CKD) patients have specific care needs that lead to the nursing professional to generate comprehensive and individualized care. For this reason, the creation of the Nephrological Unit for Continued Care (NUCA) arises in our service.

Aim: To present our experience in the implantation and development of a NUCA in the University Hospital of Henares. 
Material and Method: Retrospective descriptive study of the activity carried out at NUCA between January 1, 2017 and June 30, 2017. Development and implementation of different functions and professional competencies.

Results: In this period, a total of 804 patients were attended, with an average of 145.6 visits per month. of all these visits, 474 patients undergoing Peritoneal Dialysis ( $55 \%$ of total volume), 149 patients with CKD (16\%), 245 patients with advanced CKD (18\%) and 11 patients in conservative treatment (1\%). Different techniques were carried out, either programmed by electronic agenda or by demand.

Conclusions: The implantation of the NUCA, in a structured and planned way, is a valid and necessary alternative in our health system. It allows to ensure an integral approach to renal patients and achieve a continuity of care with lower healthcare costs.

KEYWORDS: kidney failure; chronic; daytime care; medical; nephrology nursing.

\section{Introducción}

En el momento actual, se asiste en el mundo a una verdadera epidemia de las así llamadas "enfermedades crónicas no comunicables": diabetes mellitus, hipertensión arterial, enfermedad cardiovascular y en especial, la enfermedad renal crónica (ERC). Éstas causarían actualmente el $60 \%$ de las muertes en el mundo y alrededor del $47 \%$ de los gastos en salud. Se estima que serán la principal causa de muerte y discapacidad para el 2020 y la mayor carga provocada por estas enfermedades se producirá sobre los países menos desarroIlados $^{1}$.

La situación de la ERC representa un importante problema de salud pública, tanto por su elevada incidencia y prevalencia, como por su importante morbimortalidad y su coste socioeconómico. El reconocimiento de la ERC como un problema de salud pública ha evolucionado, en parte, a partir de la aceptación del modelo conceptual, definición y clasificación de la ERC propuesta por la National Kidney Foundation, a través de la Kidney Disease Outcome Quality Initiative en 2002 y modificada por la iniciativa Kidney Disease Improving Global Outcomes (KDIGO) en 2004². En la actualidad hay evidencia convincente de que la ERC puede ser de- tectada mediante pruebas de laboratorio simples y que el tratamiento puede prevenir o retrasar las complicaciones de la función renal disminuida, retrasar la progresión de la enfermedad renal y reducir el riesgo de enfermedades cardiovasculares. Traducir estos avances a medidas simples y aplicables de salud pública debe ser adoptado como un objetivo a nivel mundial ${ }^{3}$.

A nivel de atención especializada, ofrecer una atención médica nefrológica global de calidad, centrada en el paciente, basada en la evidencia científica y costo eficiente resulta necesario. El modelo de hospital de día supone una alternativa asistencial a la hospitalización convencional, favoreciendo la continuidad asistencial y promoviendo una asistencia coordinada, ágil y ambulatoria sin los inconvenientes del ingreso ni la prolongación de la estancia hospitalaria ${ }^{4}$. Esta modalidad de atención está ampliamente implantada en algunas especialidades médicas como hematología y oncología, si bien es difícil encontrarla en el área de nefrología. Aunque existen centros donde se plantea y se acerca al concepto de hospital de día, su definición y abordaje no está ampliamente descrito ${ }^{5}$.

Desde nuestra sección de Nefrología entendíamos su potencial beneficio y planteamos su puesta en marcha y desarrollo en nuestro centro, adaptado a nuestro medio y recursos disponibles. La creación de una Unidad Nefrológica de Atención Continuada (UNAC) permite ofrecer numerosos beneficios al paciente renal, especialmente en la continuidad asistencial, en los procedimientos diagnósticos y terapéuticos, y al mismo tiempo puede reducir tiempos de hospitalización, optimizar recursos y obtener un menor coste sanitario.

Mostramos la experiencia en la creación de dicha unidad en el Hospital del Henares, que surge de la necesidad de unificar la atención a los pacientes con ERC, de alta complejidad y comorbilidad.

El objetivo del presente trabajo es presentar el proceso por el que se ha desarrollado la UNAC en nuestro centro.

\section{Material y Método}

Tras una revisión en la literatura de experiencias previas tanto en Nefrología como en otras especialidades, planteamos las distintas facetas que eran abordables en un Hospital de día Nefrológico y como adaptarlas a los recursos existentes en nuestro centro. Una vez es- 
tructuradas y definidas las actividades, analizamos los resultados de las mismas, con el objetivo de evaluar la repercusión y poder plantear posteriormente un proyecto de mejora continua.

Una vez desarrollada nuestra estructura mostramos la actividad realizada, los recursos disponibles y su aplicación, así como los resultados alcanzados en los primeros 6 meses desde su puesta en marcha. En la Tabla 1 se muestran los objetivos potenciales a desarrollar en este tipo de unidades.

Tabla 1. Objetivos de la creación de la Unidad Nefrológica de Atención Continuada (UNAC).

\section{Objetivos de la UNAC}

1. Centralizar y homogeneizar la asistencia de los pacientes con enfermedad renal crónica susceptibles de tratamiento renal sustitutivo

2. Informar, orientar y acompañar al paciente en la toma de decisión sobre las técnicas de sustitución renal

3. Coordinar la entrada en la técnica sustitutiva elegida, evitando complicaciones y uso de accesos temporales

4. Planificar la creación del acceso vascular y/o implante de catéter peritoneal

5. Evaluar las opciones de trasplante renal (vivo o cadáver)

6. Desarrollar y promocionar la atención y el cuidado del enfermo renal crónico en tratamiento conservador, no susceptible de tratamiento renal sustitutivo

7. Realizar y coordinar estudios funcionales específicos renales

8. Realizar tratamientos y procedimientos específicos de nefrología clínica

9. Participar en las actividades de prevención, promoción y conservación de la salud de una forma integral a los pacientes, con una participación activa del usuario, buscando el auto-cuidado y la independencia del paciente renal

La actividad desarrollada está basada en la participación en las actividades de prevención, promoción y conservación de la salud de una forma integral, con la búsqueda de una participación activa del usuario, facilitando el autocuidado y la independencia del paciente renal. Así, será prioritario centralizar y homogeneizar la asistencia de este tipo de pacientes, tanto de los susceptibles a tratamiento renal sustitutivo como de aquellos con indicación o decisión compartida de tratamiento médico conservador. En este sentido, uno de los aspectos clave será el acompañamiento en la toma de decisión sobre técnicas de tratamiento renal sustitutivo (TRS), junto a la aplicación de una coordinación y planificación tanto de accesos vasculares como de implante de catéteres peritoneales. La entrada de forma programada en la técnica sustitutiva elegida, sin necesidad de accesos temporales y con el menor número de complicaciones será otro aspecto primordial en esta área. Finalmente, en relación con la ERC, también se incluirá la evaluación de las opciones de trasplante renal (vivo o cadáver). A estos apartados, se incorporará de forma regular estudios funcionales y específicos renales, sin necesidad de ser ERC, y a su vez también serán parte de la actividad la administración y la realización de tratamientos y procedimientos específicos de nefrología clínica.

Para su desarrollo será necesaria una dotación mínima básica, adecuada a los objetivos planteados. Con este fin resulta imprescindible, para asegurar una adecuada atención al paciente, contar con una agenda independiente de enfermería y un espacio físico propio adecuado. Nuestra Unidad se encuentra en la primera planta del Hospital, donde se ubica un despacho de enfermería, dos boxes de uso técnico, con dotación básica (camilla, armarios, balanza, tensiómetro, panel de curas y calentador), un despacho médico y una sala dedicada al almacenaje de material (Figura 1). Esta actividad se realiza en días laborables de lunes a viernes, en horario de 8 a 22 horas, por dos enfermeras y un auxiliar de enfermería en turno de mañana y una enfermera en turno de tarde.

Una vez iniciada su actividad se realizó un análisis descriptivo retrospectivo de dicha actividad desde el 1 de enero 2017 hasta el 31 de junio 2017, mediante recogida de información de las agendas electrónicas de enfermería de nuestro centro, a través del sistema informático SELENE.

La población diana de nuestra actividad incluye pacientes con ERC en todos sus estadios: ERC con un filtrado glomerular estimado (FGE) $>30 \mathrm{ml} / \mathrm{min}$, enfermedad renal crónica avanzada (ERCA) con un $\mathrm{FGE}<30 \mathrm{ml} /$ min, tanto en tratamiento conservador como orientados a TRS y pacientes incluidos dentro del programa de diálisis peritoneal (DP). En la Tabla 2 se muestra un resumen de las actuaciones y procedimientos que se llevan a cabo en la UNAC.

\section{Actuaciones y procedimientos en paciente con ERC y FGE $>30 \mathrm{ml} / \mathrm{min}$}

En la unidad se atiende a pacientes con un FGE mayor de $30 \mathrm{ml} / \mathrm{min}$, derivados desde la consulta de Nefrología, bien de forma programada o a demanda para la realización de procedimientos, técnicas o tratamientos específicos. 

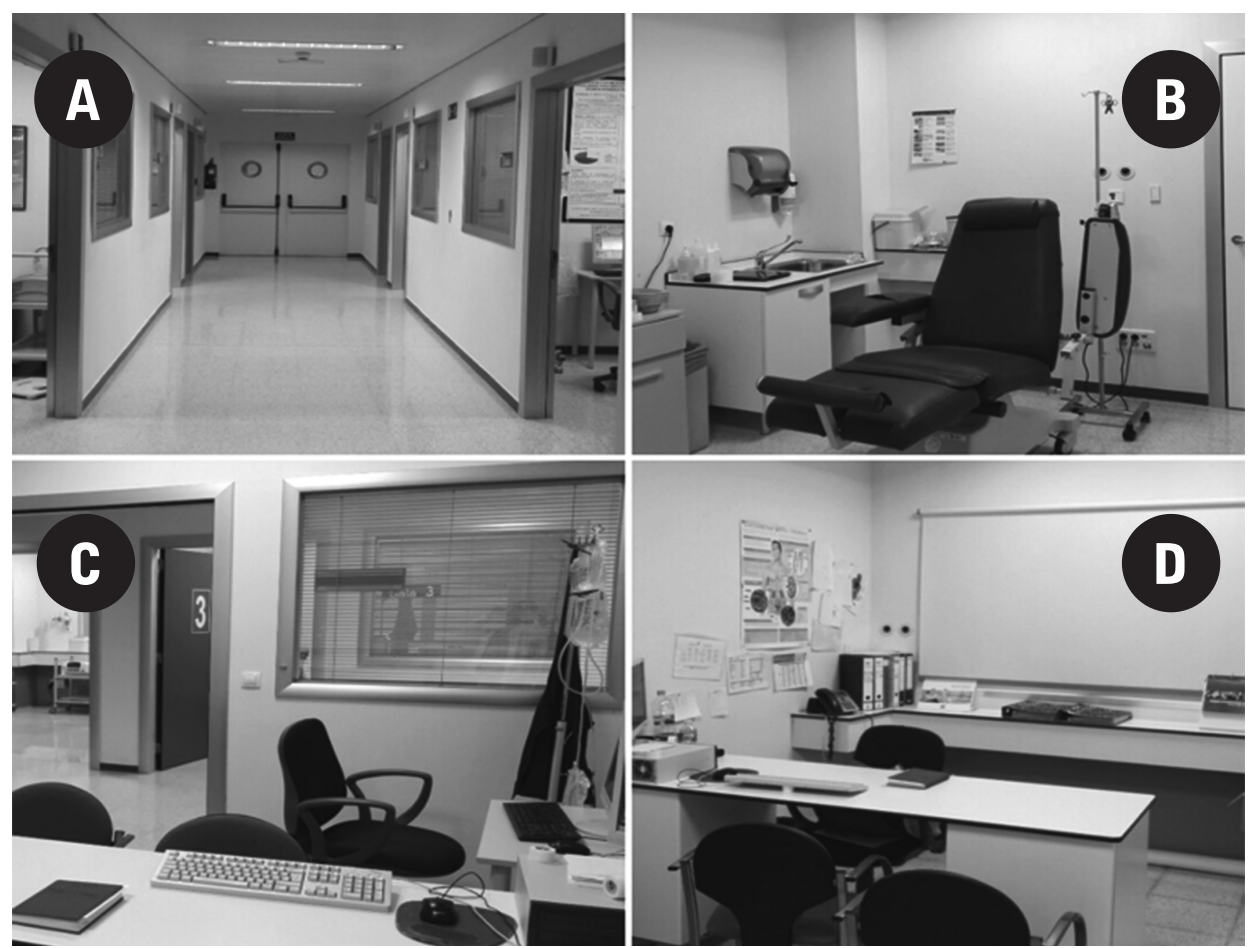

Figura 1. Dotación de la Unidad Nefrológica de Atención Continuada (UNAC). A.- Área de trabajo de la UNAC. B.- Sala de atención al paciente, realización de técnicas y entrenamientos. C y D.- Consulta de enfermería.

Estos procedimientos en población con ERC y $F G E>30 \mathrm{ml} / \mathrm{min}$ incluyen la extracción de muestra para analítica, estudios electrocardiográficos, estudio de la onda de pulso arterial, control de las vacunaciones (especialmente del virus de la hepatitis $B$, en coordinación con Atención Primaria) y los test funcionales, tanto para el estudio de los trastornos del metabolismo mineral. A su vez, también se incluyen las administraciones terapéuticas intravenosas como las encaminadas a la profilaxis (especialmente las relacionadas con el riesgo de nefrotoxicidad) o alguna antibioterapia de uso exclusivo hospitalario.

\section{Actuaciones y procedimientos en pacientes con ERCA}

En la UNAI también se atiende a pacientes con un FGE menor de $30 \mathrm{ml} /$ min, derivados de la consulta ERCA. En una primera consulta, se recoge la información para realizar una historia de enfermería que incluye un estudio antropométrico y nutricional.
Tabla 2. Actuaciones y procedimientos de la Unidad Nefrológica de Atención Continuada (UNAC), según el tipo de paciente atendido. ERC: enfermedad renal crónica con un filtrado glomerular estimado $(F G E)>30 \mathrm{ml} / \mathrm{min}$. ERCA: enfermedad renal crónica avanzada con $\mathrm{FGE}<30 \mathrm{ml} / \mathrm{min}$.
Procedimientos, técnicas y tratamientos específicos en pacientes con ERC

1. Extracciones analíticas

2. Monitorización ambulatoria de la presión arterial (MAPA)

3. Estudio electrocardiográfico

4. Vacunaciones

5.Test funcionales: sobrecarga de calcio, deshidratación,...

6. Tratamientos de administración parenteral: ferroterapia iv, antibioterapia,...

7. Profilaxis de nefrotoxicidad por contrastes
Procedimientos, técnicas y tratamientos específicos en pacientes con ERCA
1. Medición de la presión arterial
2. Medida del Índice tobillo- brazo
3. Cálculo del Índice Masa Corporal (IMC)
4. Medida de la circunferencia del brazo y di- namometría
5. Estudio nutricional
6. Evaluación del control de la anemia
7. Evaluación de la adherencia al tratamiento
8. Vacunaciones
9. Profilaxis de nefrotoxicidad por contrastes
10. Extracciones analíticas

Procedimientos, técnicas y tratamientos específicos en pacientes en DP

1. Implicación en quirófano, primer lavado y asesoramiento en tiempo real

2. Seguimiento pos-operatorio inmediato, curas, lavados y complicaciones

3. Entrenamiento de en la técnica de DP

4. Primera visita domiciliaria

5. Extracciones analíticas, protocolizadas, urgentes y cálculo de KT/V y PET

6. Controles semestrales:
- PIA
- BIA
- Exudado nasal y faríngeo
- Cambio de prolongador, bajo técnica estéril 
Esta toma de datos se repite según las necesidades de cada paciente. Los procedimientos, a parte de los ya descritos en los pacientes sin ERCA, tendrán el objetivo concreto de preservar el capital venoso. A su vez se abordan las recomendaciones nutricionales $y$ dietéticas, el cumplimiento terapéutico y las dificultades de la adherencia al tratamiento. Al igual que en el grupo anterior también se asumen las administraciones intravenosas tanto profilácticas como terapéuticas.

A medida que el FGE va disminuyendo (menor de 20 $\mathrm{ml} / \mathrm{min}$ ) se citará al paciente de forma programada en la consulta para informar sobre las distintas opciones de TRS incluso, en cumplimiento de la Ley Básica Reguladora de la Autonomía del Paciente y de Derechos y Obligaciones en Materia de información y Documentación Clínica, de aquellas que no vayan a poder elegir, explicando el motivo que lo desaconseja ${ }^{6}$.

Se exponen las alternativas actuales, como la hemodiálisis (HD) y se informa de posibilidad de tratamiento domiciliario y en hospital. También se explica la DP, con sus alternativas, diálisis peritoneal continúa ambulatoria (DPCA) y diálisis peritoneal automática (DPA) y a su vez se informa sobre trasplante renal (TR), donde se detalla la posibilidad del mismo y las distintas opciones, pudiendo ser de donante cadáver o de vivo. Finalmente, también se comenta la opción de tratamiento renal conservador en los casos pertinentes. En ocasiones, son necesarias varias visitas hasta que el paciente toma una decisión sobre la terapia a elegir. Cuando la elección es HD, se activa la creación de un acceso vascular coordinando con el centro de referencia para la realización de una imc arteriovenosa (FAV). Se le indican consejos previos al intervencionismo, métodos de potenciación mediante ejercicios y recomendaciones sobre cómo evitar punciones innecesarias. Se realiza una valoración del acceso vascular post-intervención precoz (en 24 horas) en la UNAC, donde se dan las recomendaciones para el mantenimiento de la FAV y se realiza un seguimiento de la misma, que incluye: cuidados y retirada de puntos, seguimiento de la maduración de la FAV, detección precoz de problemas y mantenimiento hasta su utilización definitiva. En la segunda visita, se realiza un primer control ecográfico de la $\mathrm{FAV}$, con el objetivo de verificar su desarrollo y características de la misma. Posteriormente, se realiza un seguimiento del estado de la FAV en cada visita que el paciente realiza en la consulta hasta el momento de su entrada en HD.

\section{Actuaciones y procedimientos en pacientes inclui- dos en programa de DP}

Si el paciente opta por DP se le guía al igual que en otras técnicas. Se incluye la coordinación con el servicio responsable del implante del catéter peritoneal (CP), en nuestro centro, el Servicio de Cirugía General.

Una vez implantado el CP se realiza el seguimiento de su evolución durante el periodo de cicatrización, mediante revisiones semanales, con lavado y cura del catéter, y valoración de posibles complicaciones del mismo. Dependiendo de los síntomas y signos clínicos y de los resultados analíticos, se comienza el entrenamiento entre la tercera y cuarta semana de la implantación del catéter, con el objetivo de completar el periodo de cicatrización y llegar con el paciente correctamente entrenado en la técnica. A partir de ese momento se coordina el envío del material necesario para iniciar la técnica en su domicilio, cuando se estime oportuno. El inicio de DP en domicilio incluye una primera visita de un profesional de enfermería para supervisar el primer intercambio del paciente. En nuestro centro, durante el entrenamiento se determina la presión intraabdominal (PIA) con el fin de adecuar la técnica de forma individualizada. En los controles y seguimientos protocolizados en DP se realiza un control a los quince días de inicio de TRS, para adecuar la técnica de diálisis y posteriormente bimensuales. EI personal de enfermería realiza las analíticas indicadas junto a los procedimientos necesarios para el cálculo del KTV y TEP (test de equilibrio peritoneal) o PET (Peritoneal equilibrius test). También se incluye la recogida de exudado nasal y faríngeo semestralmente, junto con una medición de bioimpedancia eléctrica (BIA). Hasta que se estime oportuno el inicio de TRS el paciente acude semanalmente para practicar un lavado y la cura del orificio de salida del catéter.

\section{Resultados}

Desde Enero a Junio de 2017, en nuestra Unidad se han realizado un total de 874 visitas, con una media de 145,6 visitas al mes. Por tipo de pacientes atendido el $55 \%$ fueron de DP, $28 \%$ de ERCA, $1 \%$ tratamiento conservador y $16 \%$ a otros (pacientes con ERC y $\mathrm{FGE}>30 \mathrm{ml} / \mathrm{min}$ ).

De forma detallada en el área de diálisis peritoneal se realizaron 474 visitas durante el periodo de estudio. Se realizaron diferentes técnicas, todas ellas programadas mediante las agendas electrónicas, o bien a demanda del nefrólogo. Las diferentes técnicas que se han regis- 
trado fueron mayoritariamente el pase en la consulta conjunta con el nefrólogo con 85 visitas. También se realizaron 15 entrenamientos de pacientes nuevos, que precisaron 72 días hasta tener adquiridos los conocimientos necesarios lo que supone una media de entrenamiento por paciente de 4,8 días, 88 cálculos de Kt/ $\mathrm{V}$ con su correspondiente analítica, 29 test de equilibrio peritoneal (PET), 11 mediciones de presión intraabdominal (PIA), 98 intervenciones de atención al paciente peritoneal, donde englobamos lavados peritoneales de pacientes que están en descanso o a la espera de entrar en técnica, intercambios peritoneales y curas del orificio de salida. Otras visitas que se han contabilizado de forma general representan: 5 comprobaciones de catéteres en quirófano, 2 visitas domiciliarias, 28 curas en general, 3 intervenciones ante peritonitis, 4 ingresos, 7 vacunas ante el virus de hepatitis $B, 5$ mediciones de presión arterial ambulatoria (MAPA), 5 administraciones de hierro intravenoso, 8 administraciones de diferentes antibióticos y 13 determinaciones de anticuerpos antilinfocitotóxicos.

En el área de ERCA se realizaron 245 visitas en este periodo, bien citadas de forma programada mediante la agenda electrónica o bien a demanda del nefrólogo. Se realizaron 158 analíticas, haciendo hincapié en el cuidado del árbol vascular. Se incluyeron también 10 primeras visitas donde el paciente acude para valoración inicial en la UNAC, 12 consultas sucesivas por necesidades del paciente, 13 explicaciones de las diferentes técnicas de sustitución renal, 7 revisiones de FAV de pacientes en descanso o prediálisis. A su vez se contabilizaron en el apartado de otros, 19 visitas para vacunación de la hepatitis $B, 4$ MAPAs, 13 administraciones de hierro intravenoso, 6 administraciones de diferentes medicaciones y 3 realizaciones de electrocardiograma.

En el grupo de pacientes no en DP, ni ERCA, pero con $E R C$ con $F G>30 \mathrm{ml} / \mathrm{min}$. Se realizaron 149 visitas. En 89 de las visitas se realizaron extracciones analíticas $(59,73 \%)$, en 23 visitas se procedió a la colocación de medición de presión arterial ambulatoria, en 15 visitas se administró la vacuna frente a la hepatitis $B$, en 9 se administró tratamiento con hierro intravenoso, en un caso se realizó un electrocardiograma, en 5 ocasiones se llevó a cabo un estudio de la onda de pulso arterial y en 7 ocasiones se realizó un test de sobrecarga oral de calcio y en otro caso un test de deshidratación.

En el apartado de atención al paciente en tratamiento conservador, donde se engloban pacientes que no van a ser incluidos en técnica de sustitución renal, se realiza- ron analíticas de seguimiento $(n=30)$ y asesoramiento de cuidados paliativos $(n=7)$.

\section{Discusión}

Según el Real Decreto 1277/20031, una unidad de hospitalización de día se define como "unidad asistencial donde, bajo la supervisión o indicación de un médico especialista, se lleva a cabo el tratamiento o los cuidados de enfermos que deben ser sometidos a métodos de diagnóstico o tratamiento que requieran durante unas horas atención continuada médica o de enfermería, pero no el internamiento en el hospital ${ }^{\prime \prime 7}$.

Este concepto se ha desarrollado en diversas especialidades con resultados positivos. En nefrología, aunque es conocido que existen centros con una estructura semejante, la definición de sus competencias, así como la organización del mismo no está ampliamente descrita. A pesar de la escasa documentación publicada, la experiencia descrita en el servicio de Nefrología del Hospital de Puerto Real (Cádiz) en el año 2011 es de las pioneras en nuestro país, con resultados positivos tanto para el paciente como para los profesionales ${ }^{5}$. Recientemente la iniciativa del Hospital Ramón y Cajal de Madrid también, con una estructura consolida$\mathrm{da}$, ha mostrado los mismos resultados favorables, que ponen en evidencia los potenciales beneficios ${ }^{8}$.

Comparativamente otras especialidades también han desarrollado con éxito este modelo de hospital de día. Así, en el Hospital del Mar de Barcelona, se ha descrito un modelo de atención semejante en el servicio de Neumología, donde evidenciaron su utilidad en la gestión de la atención a pacientes con patología respiratoria, con la reducción de las necesidades de hospitalización, pero manteniendo la calidad asistencial'. El Hospital General Universitario de Ciudad Real, describió un hospital de día médico en el área reumatológica, ofreciendo una asistencia multidisciplinaria en un ambiente hospitalario y realizando labores de diagnóstico, exploraciones complementarias y tratamientos, que por su particularidad no se podían realizar en la consulta externa ni justificaban un ingreso hospitalario ${ }^{10}$.

Otra experiencia mostrada por el servicio de aparato digestivo del Parc Taulí en Sabadell, donde se cambiaba el modelo previo centrado en la atención del paciente en la sala de hospitalización, a otro con un manejo ambulatorio, sin ingreso hospitalario, fue muy 
favorable en la reducción de ingresos y en el control de pacientes de elevada complejidad ${ }^{11}$.

En resumen, los hospitales de día, como alternativas a la hospitalización convencional, son una necesidad asistencial, que ha quedado puesto de manifiesto en las experiencias descritas, así como en otras más clásicas como Oncología o Hematología. Posteriormente se ha ido extendiendo a otros ámbitos (enfermedades psiquiátricas, enfermos de SIDA, pacientes pluripatológicos, etc. ${ }^{12}$, con un considerable desarrollo durante los últimos años, que se ha traducido, al menos, en una reducción de los pacientes ingresados en unidades de hospitalización convencional ${ }^{13}$.

Este tipo de atención continuada sin hospitalización promueve un cambio cultural en la asistencia, que permite optimizar los recursos del hospital para diagnóstico y tratamiento, más racional y ágil, favoreciendo mantener al enfermo en su entorno habitual ${ }^{14}$. Apenas existen estudios que evalúen la atención médica del paciente en los hospitales de día de Nefrología en España. La nefrología es una especialidad que, por sus características técnicas, se beneficiaría ampliamente de este tipo de modelo de atención. El desarrollo del hospital de día en el servicio de nefrología puede contribuir a potenciar la autonomía de atención especializada en este tipo de pacientes ${ }^{5}$. Pocas especialidades médicas disponen de tantos procedimientos y técnicas especiales, por lo que su desarrollo no solo es recomendable sino necesario dada la prevalencia de la enfermedad renal crónica, todavía más si cabe con el envejecimiento poblacional al que debemos enfrentarnos. A su vez desde la Sociedad Española de Nefrología (SEN) se ha propuesto su desarrollo como pieza clave de la atención al enfermo renal y como incentivación profesional, haciendo especial hincapié en la nefrología intervencionista ${ }^{15,16}$. Asumir aspectos relacionados con el acceso vascular y peritoneal, así como con el diagnóstico y las técnicas ecográficas, potenciar la gestión clínica y la resolución ambulatoria, siempre bajo una mínima estancia, puede ser clave para la sostenibilidad del sistema ${ }^{17}$.

Nuestro objetivo es mostrar el proceso de su desarrollo en nuestro centro, con la creación de una unidad de nefrología de atención continuada (UNAC), como proyecto de mejora continua, con unas líneas estratégicas fundamentales, optimizando los recursos y las estructuras disponibles.

Sobre nuestros pacientes con ERC, potencialmente podemos observar beneficios específicos en los distintos apartados. De forma individualizada, algunas pruebas, como la MAPA; va a permitir una aproximación a la PA real y a los riesgos relacionados, más exacta que la derivada de la PA en la consulta. Su desarrollo en la UNAC supone un mayor control y un abordaje precoz de este factor de riesgo. Las anomalías en la PA ambulatoria, especialmente las observadas en la PA sistólica y en la PA nocturna, están estrechamente relacionadas con el riesgo cardiorrenal del paciente con ERC, por lo que su control justifica un uso más amplio de la MAPA en pacientes renales ${ }^{18}$. Respecto al control de las vacunaciones, conocer la serología completa del paciente antes de llegar a la técnica elegida supondrá optimizar los beneficios de la misma y asegurarse un correcto cumplimiento de las pautas de vacunación. Su seguimiento permitirá evaluar las necesidades de revacunaciones y de nuevas administraciones según se desarrollen necesidades. A su vez, la administración de formulaciones iv, como la ferroterapia iv, permitirá un control mayor de la anemia renal optimizando los agentes estimuladores de la eritropoyesis y asegurando el correcto cumplimiento terapéutico. En estos casos la administración de hierro intravenoso es esencial para asegurar la efectividad del tratamiento de la anemia de causa renal y favorecer la respuesta al tratamiento ${ }^{19}$.

Sobre nuestros pacientes con ERCA, existen otros beneficios en esta población, con algunas pruebas específicas como el índice tobillo-brazo o el análisis de la onda de pulso arterial (arteriograph), que nos permitirá detectar el paciente de mayor riesgo con calcificación de rigidez a nivel del árbol vascular ${ }^{20}$. A su vez el mayor conocimiento de la situación del paciente permitirá incidir en aspectos terapéuticos. El conocimiento de los mismos, con índice Masa Corporal, el perímetro de cintura, el estado nutricional, la circunferencia del brazo y la dinamometría son necesarios para un seguimiento periódico por parte de enfermería, donde se informe y se recalque la importancia del autocuidado ${ }^{21,22}$. Al mismo tiempo es clave la educación en los fármacos indicados, importancia de los mismos, administración adecuada y potenciales efectos secundarios para obtener una idónea adherencia al tratamiento ${ }^{23}$. También será clave el conocimiento de la ERC que padece y los potenciales riesgos ante determinados fármacos y pruebas, especialmente en las pruebas con contraste radiológico. La profilaxis en el uso de contrastes yodados será determinante en muchos casos para preservar la función renal24. En la línea de la prevención, la importancia de velar al máximo por el árbol vascular cara a la realización de un acceso arterio-venoso, condicionará la mejor evolución a largo plazo ${ }^{25}$. 
Respecto a los beneficios en nuestros pacientes que están en técnica renal sustitutiva diálisis peritoneal (DP), la presencia física en quirófano para la comprobación del catéter in situ antes de que el cirujano proceda a cerrar incisión, el entrenamiento del paciente pasado el periodo de cicatrización, la visita al domicilio del profesional de enfermería para supervisar el primer intercambio del paciente son aspectos a destacar. A parte de los controles protocolizados, la medición de la presión intraabdominal (PIA) ${ }^{26}$ como práctica rutinaria va a permitir diferenciar perfiles de pacientes para individualizar su tratamiento. También la realización de forma sistemática de bioimpedancia eléctrica (BIA) va a permitir adecuar el volumen de infusión del líquido peritoneal para individualizar su tratamiento, analizar la composición corporal y el estado de hidratación de forma objetiva ${ }^{27}$. Una sobrehidratación continuada está asociada con morbilidad y mortalidad de los pacientes en diálisis y su control podría mejorar dicha condición ${ }^{28}$.

Finalmente, es clave destacar la trascendencia de la capacitación del profesional sanitario. Dichas experiencias se basan en una mayor autonomía y capacidad de decisiones de diferentes profesionales implicados en la atención del enfermo renal. En este sentido el papel de enfermería es clave para la correcta funcionalidad de estas áreas, asumiendo mayor responsabilidad, basada en un mayor conocimiento e independencia, sustentada en un trabajo colectivo multidisciplinar ${ }^{29}$.

\section{Conclusiones}

La puesta en marcha de una unidad de atención especializada en nefrología, sin necesidad de hospitalización es una alternativa válida para los pacientes renales. El desarrollo de una atención integral en nefrología sin hospitalización, amplía la posibilidad de un seguimiento especializado y específico. Su aplicación de una forma estructurada, basada en una autoevaluación y necesidades progresivas es necesaria en el sistema actual de salud. La implicación del profesional especializado será un aspecto clave para optimizar la atención de estos pacientes de alta complejidad, con el consiguiente beneficio para los mismos, y poder ofrecer una mayor individualización del tratamiento y un mayor autocuidado, basando el abordaje de la enfermedad en el propio paciente.

Aunque nuestros datos son preliminares y mostramos fundamentalmente la actividad de seis meses, los potenciales beneficios y su desarrollo pueden aportar una información no ampliamente desarrollada y ser un pun- to y seguido en su aplicación de una forma más sistemática en los centros hospitalarios.

\section{El autor declara que no hay conflicto de interés.}

Recibido: 7 diciembre 2017

Revisado: 25 enero 2018

Modificado: 2 febrero 2018

Aceptado: 15 febrero 2018

\section{Bibliografía}

1. Yach D, Hawkes C, Gould L, Hofman K. The Global Burden of Chronic Diseases. Overcoming Impediments to Prevention and Control. JAMA 2004; 291:2616-22.

2. National Kidney Foundation. K/DOQI clinical practice guidelines for chronic kidney disease: evaluation, classification and stratification. Am J Kidney Dis 2002; 39(Supl 1):S1-266.

3. Levey AS, Atkins R, Coresh J, Cohen EP, Collins AJ, Eckardt KU et al. Chronic kidney disease as a global public health problem: approaches and initiatives - a position statement from Kidney Disease Improving Global Outcomes. Kidney Int. 2007; 72(3):247-59.

4. Hernando Ortiz L, Hinojosa Mena-Bernal C, González Sarmiento E, González Guilabert I, Arana Ruiz $J$, Muñoz Moreno MF. Rentabilidad de un hospital de día: análisis de actividad, coste y eficacia. Gac Sanit. 2012; 26(4):360-5.

5. Remón Rodríguez C, Quirós Ganga PL, González-Outón J, del Castillo Gámez R, García Herrera AL, Sánchez Márquez MG. Recuperando actividad e ilusión: el hospital de día médico de nefrología. Nefrologia. 2011;31(5):545-59.

6. Pastor JL, Julián JC. Claves del proceso de información y elección de modalidad de diálisis en pacientes con insuficiencia renal crónica. Nefrologia 2010;1(Supl Ext 1):S15-20. 
7. Ministerio de Sanidad y Consumo. Dirección General Agencia de Calidad del SNS. Plan de Calidad para el Sistema Nacional de Salud 2006. [Internet] Madrid: Ministerio de Sanidad y Consumo; 2007 [consultado 28 abril 2011]. Disponible en: http://www.msc.es/organizacion/sns/planCalidadSNS/pncalidad.htm

8. Sosa Barrios RH, Burguesa Vion V, Chacón E, Chediak Terán C, Campillo Trapero C et al. Hospital de día Nefrológico (HdD): Experiencia en un centro de tercer nivel. Libro de Abstracts y Casos de la XIII Reunión de la Sociedad Madrileña de Nefrología. Madrid: Sociedad Madrileña de Nefrología; 2017.

9. Cots F, Raventòs J, Ausín P, Chiarello P, Balcells E, Castells X, Gea J. Hospital de día: análisis de resultados, costes y asignación de recursos en neumología. Arch Bronconeumol. 2013;49(2):54-62.

10. Ramírez Huaranga MA, Arenal López R, Parraga Prieto C, Anino Fernández J, Minguez Sánchez $M D$ et al. Importancia del hospital de día en Reumatología. Apunt. Cienc. 2013;3 (Extraordinario):230-231.

11. Vergara Gómez M, Gil Pradesa M, Dalmau Obrador B, Miquel Planas M, Sánchez Delgado J, Calvet Calvo $X$ et al. Unidad de atención continuada y hospital de día como alternativa a la hospitalización convencional: experiencia de 10 años en un hospital comarcal. Gastroenterol Hepatol 2007; 30 (10):572-9.

12. Sauret Valet J. Hospitales de día ¿generales o especializados? Arch Bronconeumol 1994;30:477-8.

13. Román Ivorra JA, Gómez-Salazar JR, Calvo Catalá J. Estado actual de los hospitales de día donde se administran los tratamientos de reumatología en la Comunidad de Valencia. Reumatol Clin. 2010; 6(5):244-9.

14. Torres Salinas M, Capdevila Morel JA, Armario García P, Montull Morer S. Alternativas a la hospitalización convencional en medicina interna. Med Clin (Barc) 2005;124(16):620-6.

15. Sociedad Española de Nefrología. El libro blanco de la Nefrología española. Nefrologia 2000; 20(5):396-402.
16. Rivera M, Quereda C. Nefrología diagnóstica e intervencionista: una oportunidad para los nefrólogos españoles. Nefrologia 2011;31(2):131-3.

17. De Francisco ALM. Sostenibilidad y equidad del tratamiento sustitutivo de la función renal en España. Nefrologia 2011;31(3):241-6.

18. Gorostidi M, Fernández Fresnedo G, Galcerán JM, Segura J. Monitorización ambulatoria de la presión en la enfermedad renal crónica. Nefrología 2009; 29(Supl Ext. 5):S123-30.

19. Donado E, Aguasca M, Ocharan-Corcuera J, Minguela I, Gimeno I, Chena A et al. Hierro sacarosa intravenoso en el paciente con enfermedad renal. Dial Traspl. 2007;28(1):17-25.

20. Arévalo Manso JJ, Juárez Martín B, Gala Chacón E, Rodríguez Martínez C. El índice tobillo-brazo como predictor de mortalidad vascular. Gerokomos. 2012;23(2):88-91.

21. Ortega Pérez de Villar L, Antolí García S, Lidón Pérez MJ, Amer Cuenca JJ, Martínez Gramage J, Segura Ortí E. Cuantificación del deterioro funcional durante seis meses en pacientes renales en estadio terminal. Enferm Nefrol. 2015;18(4):265-71.

22. Ravasco $P$, Anderson $H$, Mardones F. Métodos de valoración del estado nutricional. Nutr. Hosp. 2010; 25(Supl 3):S57-66.

23. Nogués Solán X, Sorli Redó ML, Villar García J. Instrumentos de medida de adherencia al tratamiento. An. Med. Interna (Madrid). 2007;24(3): 138-41.

24. Gorostidi $M$, Santamaría $R$, Alcázar R, Fernández-Fresnedo G, Galcerán JM, Goicoechea M et al. Documento de la Sociedad Española de Nefrología sobre las guías KDIG0 para la evaluación y el tratamiento de la enfermedad renal crónica. Nefrología 2014;34(3):302-16.

25. Ibeas J, Roca-Tey R, Vallespín J, Moreno T, Moñux G, Martí-Monrós A, et al. Guía Clínica Española del Acceso Vascular para Hemodiálisis. Nefrología. 2017;37(Supl 1):S1-191.

26. Blasco Cabañas $C$, Ponz Clemente $E$, Betancourt Castellanos L, Otero López S, Marquina Parra D, 
Gran Pueyo $C$ et al. Relación entre la presión intrabdominal en diálisis peritoneal con las hernias y fugas. Enferm Nefrol 2012;15(2):94-100.

27. Mendías Benítez $C$, Alonso de Porras $L$, Barcia García J, Sánchez Oliva JM, Jiménez Quintana E, Ruiz Alfonso $L$ et al. Bioimpedancia eléctrica: Diferentes métodos de evaluación del estado nutricional en un centro periférico de hemodiálisis. Rev Soc Esp Enferm Nefrol. 2008;11(3):173-7.

28. Arias M. La bioimpedancia como valoración del peso seco y del estado de hidratación. Dial Traspl 2010;31(4):137-9.

29. Fishbane S, Agoritsas S, Bellucci A, Halinski C, Shah HH, Sakhiya V, Balsam L. Augmented Nurse Care Management in CKD Stages 4 to 5: A Randomized Trial. Am J Kidney Dis. 2017;70(4):498505.

Este artículo se distribuye bajo una Licencia Creative Commons Atribución-NoComercial 4.0 Internacional. https://creativecommons.org/licenses/by-nc/4.0/

\section{Open Access (C) $\underset{\mathrm{BY}}{\mathrm{N}} \mathrm{S}$}

